\title{
EXPLORATORY STUDY EXAMINING THE FEASIBILITY OF A TECHNOLOGY-BASED TOOL TO SUPPORT CREATIVITY IN CHILDREN WITH AUTISM
}

\author{
Aurora Constantin, Cristina Adriana Alexandru, Georgia Fox and Lauren Meikle \\ University of Edinburgh, School of Informatics \\ 10 Crichton Street \\ Edinburgh $E H 89 A B, U K$
}

\begin{abstract}
Previous research suggests that Participatory Design (PD) is a very promising approach for involving children with Autism Spectrum Conditions (ASC) in generating design solutions, but also in practising essential skills (e.g. collaboration skills). However, because of their difficulties with social communication accompanied by rigidity in thought and behaviour, these children face significant challenges during PD. Specifically, in the idea generation stage of PD, they have difficulties expressing their creativity. Based on user participation, we built a first version of a low fidelity prototype (iGeneration) for a technology-based tool to elicit creativity in children with ASC during idea generation. This article reports on a research study which explored the feasibility of iGeneration to support children with ASC in creating design ideas for an educational game. The results revealed that the participants engaged and enjoyed working with iGeneration. The children created a number of ideas which were diverse, novel and appropriate for the design tasks.
\end{abstract}

\section{KEYWORDS}

Participatory Design, Children, Autism, Feasibility Study, Idea Generation

\section{INTRODUCTION}

Previous research suggests that PD is a very promising approach for involving individuals with Autism Spectrum Conditions (ASC) in generating technological design solutions (Constantin et al. 2019, Escobedo et al. 2014, Grawemeyer et al. 2012, Keay-Bright. 2007). For children with ASC, PD can not only lead to the design of effective systems, but also be engaging, motivating and inspire them to become design partners (Mazzone et al. 2012). Participation is empowering, giving them confidence to share opinions and ideas with others. In addition, it offers opportunities to develop new skills, such as problem solving, collaboration (Malinverni et al. 2014), design related skills, and a reflective viewpoint towards technology, which are essential in our digital society (Iversen et al. 2017). Particularly, PD is fundamentally intertwined with creativity and could contribute essentially to unlocking it (Makhaeva et al. 2016).

Despite these widely recognized advantages, involving children with ASC in PD is often avoided because of various challenges, including their "communication barriers" (De Leo and Leroy 2008, Keay-Bright 2007) or "time and resource constraints" (Randolph and Eronen 2007). Idea generation, one of the most common PD activities, is particularly challenging as children with autism have difficulties expressing their creativity. Moreover, they require tailored support to be able to generate and communicate their design ideas.

Technology may be a good candidate to support children with ASC during the PD process. Over the last few decades, it has been increasingly designed and employed with children with ASC (Malinverni et al. 2014, Escobedo et al. 2014, Grawemeyer et al. 2012, Frauenberger et al. 2013), particularly due to their affinity toward it, as well as the advantages it brings over traditional methods (e.g. pencil and paper). However, very little has been done to explore how technology can best support children with ASC and designers/researchers during PD. Therefore, the proposed project aims to design the first personalisable technology-based tool (iGeneration) to support creativity during the idea generation stage of PD with children with ASC. We believe that such a tool can act as an interface between designers and children with ASC, making their collaboration easier within a more predictable and controllable environment compared to traditional approaches to PD. 
Moreover, technology affordances can support personalisation, making it easier for the designers/researchers to tailor their PD methods to the individual needs of the involved children.

In this paper, we describe a first low-fidelity version of the iGeneration prototype which was focused on individual interaction to generate design ideas. In the feasibility study reported, we explored whether children with ASC engage, enjoy working with the prototype, and are able to produce design ideas as supported by it and the designer only (collaboration between children will be considered in the next prototype version). To this end, we decided to use paper-based prototype screens and a researcher playing the role of the computer, instead of a technological solution. This may be seen as a limitation, but had a strong motivation given by the need to provide something tangible and concrete enough for the autistic children, to get their views on feasibility, before delving into detailed design. In particular, as they may have difficulties interpreting abstractions (Ropar and Peebles 2007) which are inevitably used in technological tools, we considered it essential to understand whether this would be an issue with our prototype. This way, we can ensure that what is being developed gets the autistic children's 'seal of approval' by respecting their needs.

This study contributed to answer the following three research questions: 1 . Could a low-fidelity prototype for technology-based tool support engagement in children with ASC during the idea generation stage of a PD process? 2. Do children with ASC enjoy using the prototype? 3. Are children with ASC able to generate design ideas using the prototype? Additionally, we were interested in collecting ideas to improve the tool.

\section{STUDIES TO INFORM THE DESIGN OF IGENERATION}

The version of the iGeneration low-fidelity prototype presented here was iteratively designed by involving various stakeholders $(\mathrm{N}=15)$ : practitioners (e.g. teachers), carers, and researchers. A description of the studies is available in (Constantin and Hourcade 2018). Once the prototype was ready, we evaluated its usability and suitability with five neurotypical (NT) children aged 7 to 11 . The study revealed that the NT children engaged and enjoyed using the prototype and that the designed activities/tasks were suitable. They also perceived the tool as being useful to support them in creating design ideas. Some usability problems were identified (e.g. visibility of some buttons and use of error prevention messages), which we addressed.

\section{THE IGENERATION PROTOTYPE}

The prototype opening page (Figure 1) welcomes the user via a customisable virtual character (VC). The child is asked to type their name, then selects a project to work on and the $\mathrm{VC}$ introduces them to the design problem through a fictional story (as in Fails et al. 2013) (Figure 2). The child can read the story or choose the VC to read the story for them. After that, the VC presents the first task, encouraging the child to generate as many design ideas as possible. It tells the child to add an idea on a digital sticky note which can be displayed when pressing the button "Add note" in the bottom left corner of the screen (Figure 3). When the sticky note is displayed, the $\mathrm{VC}$ guides the child to introduce ideas by typing, drawing, adding an image from the gallery or through voice recording. These were chosen to encourage the children's creativity and offer freedom to express themselves in their preferred way. When the child is stuck, the VC prompts them to ask for help by pressing a button, which displays hints (Figure 4). The VC rewards the child for each achievement (after adding a new idea like in Figure 5, completing a task or completing the entire project). The reward is incremental, reflecting the child's progress, as recommended by (Constantin et al. 2017) based on their research with autistic children. The designer can offer additional support during the interaction, and is meant to analyse the text, drawings, images, recordings of the child's ideas. 


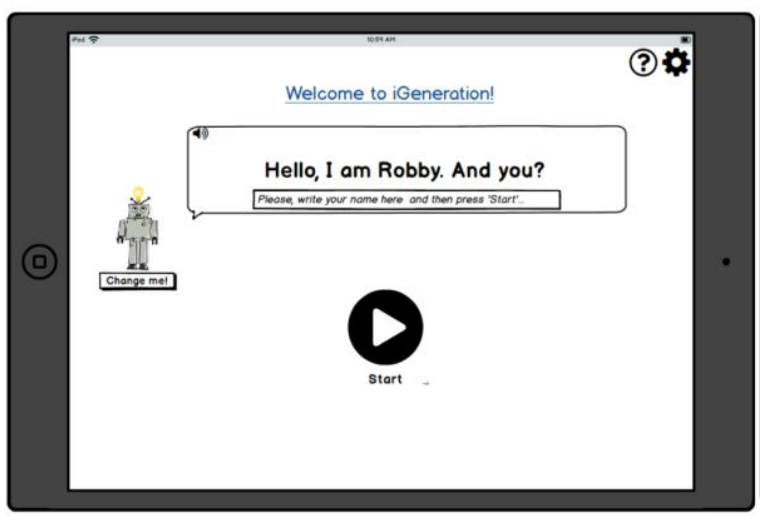

Figure 1. iGeneration: Screenshot of the opening page

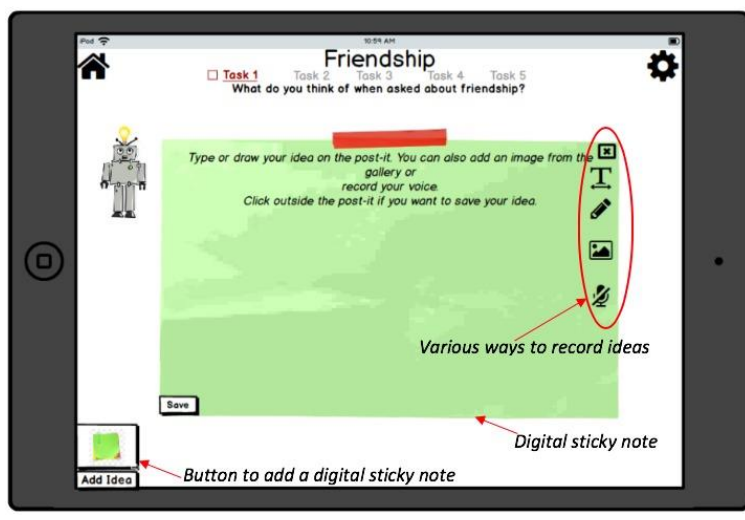

Figure 3. iGeneration: Screenshot with a sticky note

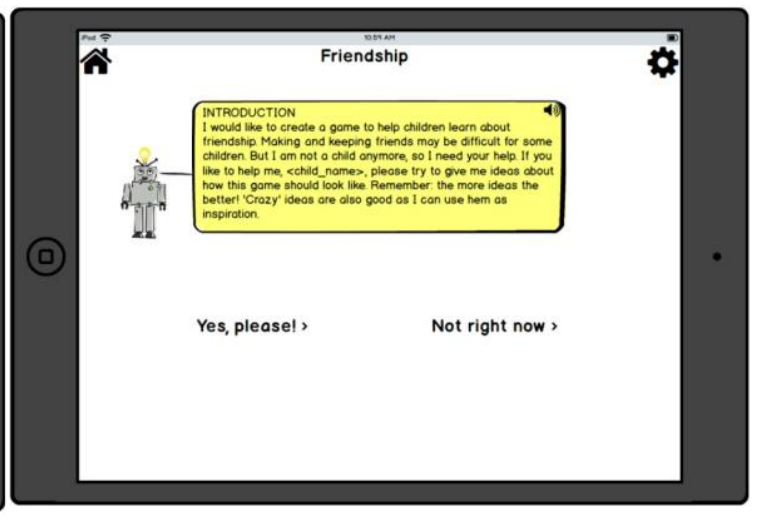

Figure 2. iGeneration: Screenshot with a story

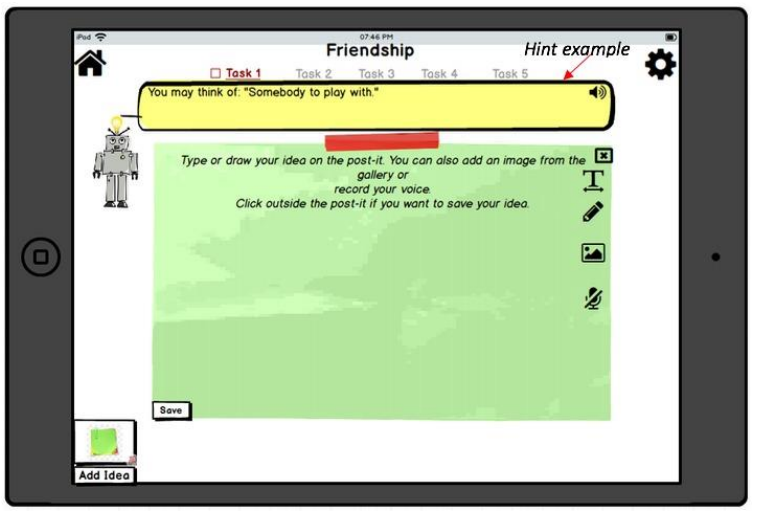

Figure 4. iGeneration: Screenshot with a hint suggested

\section{DESIGN OF THE FEASIBILITY STUDY}

The feasibility study aimed to explore whether children with ASC can interact with the prototype and are able to produce design ideas. It was carried out during a visit to a holiday-time event for autistic children organised in the Connolly School Campus in Blackburn, UK by the Lothian Autistic Society, with the researchers being offered by the organisation 4 hours to finalise it. The study was approved by the University of Edinburgh School of Education Ethics Committee. Well in advance of the day of the study, information sheets and consent forms were sent to parents. We set up the study after we received the signed consent forms. Five children (3 male, 2 female) with a previous ASC diagnosis (all at level 2 according to DSM-5 (APA, 2013)), aged 10-14 ( (Mean =12.2; SD = 1.64) and no reported comorbidities (including no intellectual disability), all verbal, participated in the study. Before beginning a session, each child provided informed assent for participation. Three children participated one-by-one, while two preferred to interact with the prototype simultaneously, yet still individually. Each child was accompanied by a parent or carer who were asked to intervene only if needed, to help explain or support the child. Three researchers were present, playing the roles of facilitator, computer to change the paper screens for the child, and video recorder of the session. A session lasted for about 40 minutes.

After a brief introduction, the children were asked to use the prototype without training and create design ideas for an educational game focused on friendship (characterising friends and the activities they would enjoy together). The game was structured around five tasks. The first four were designed to gather ideas of game features (e.g. characters and activities) while the last collected ideas of rewards (possibly different to the ones already received) children preferred when they came up with an idea, completed a task or the entire game. Finally, a short questionnaire gathered the children's perceived experience with the prototype. 


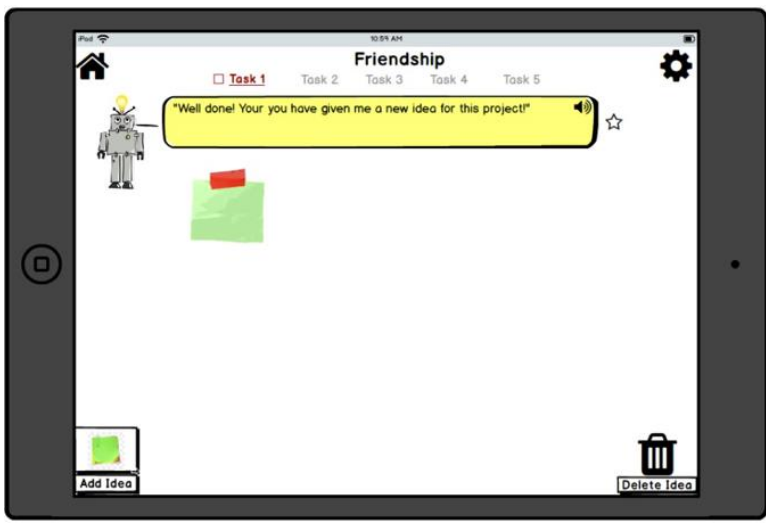

Figure 5. iGeneration: Rewards

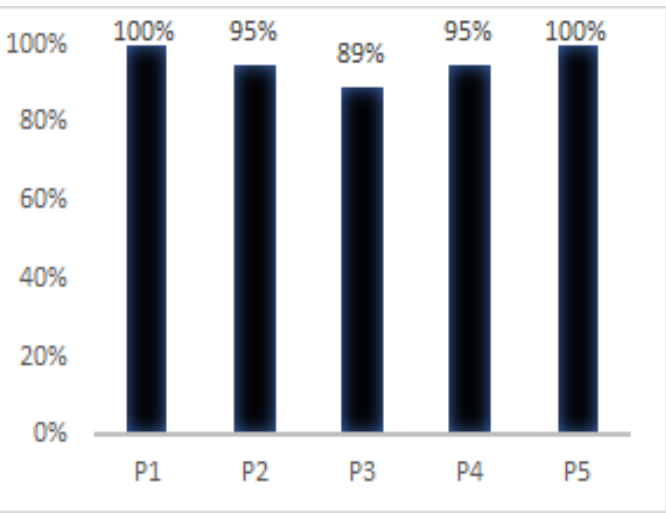

Figure 6. Levels of engagement

\section{DATA COLLECTION AND ANALYSIS}

The video recordings ( $\mathrm{N}=4, \mathrm{~T}=212$ minutes) of the sessions were transcribed (including noting non-verbal communication and pauses) two at a time by two researchers, after which they were swapped and proofread. Analysis was then performed following a top-down thematic analysis (Braun and Clarke 2006). Similarly, the researchers analysed transcripts two at a time, and swapped. Discrepancies were discussed with the team.

Similar to previous work (e.g. (Xie et al. 2008)) the level of engagement was measured by percentage of on-task time per child. The level of enjoyment during prototype use was also analysed, using three measures: 1) positive and negative verbal and non-verbal behaviours from the video recordings (Xie et al. 2008); 2) general level of child enjoyment, on a 5-point smileyometer, a Likert scale with emoticons ranging from very unhappy to very happy (Read and MacFarlane 2006; Frauenberger et al. 2013); 3) level of enjoyment with the $\mathrm{VC}$, the ways that ideas can be entered (text, drawings, images and voice recording) and the received rewards; these were also ranked using a smileyometer. To answer the third research question, all ideas generated by children were counted and analysed in terms of appropriateness (i.e. relevance to the design task), diversity/uniqueness (i.e. not being mentioned before), and novelty (not being facilitated by a hint).

\section{RESULTS}

Level of engagement. The time spent by the children on-task was $96 \%$ of the total time across the four sessions, and is represented for each child in percentages in Figure 6. Breaks were not requested. Moreover, three children were asked to move to the next task after generating a number of ideas, due to our time limit.

Level of enjoyment. On the general opinion smileyometer, three children selected an emoticon for 'very happy', one for 'happy' and one for 'neither happy nor unhappy'. In addition, the analysis of verbal and non-verbal behaviour revealed a number of instances when children enjoyed working with the prototype, and no frustration with it (Figure 7). All children reported they liked the virtual character (2 selected 'very happy' and 3 'happy'). Most of them liked the ways of recording ideas and the rewards, with no answer lower than 'neither happy nor unhappy' but some variability (especially for the ways of recording ideas).

Ideas Generated. Children generated 39 ideas, with at least one per task per child (Figure 8). The maximum number of ideas generated by a child across tasks was 10 . However, the children did not exhaust their ideas since a time limit was imposed. Only two employed all ways of introducing their ideas. Out of the 39 ideas generated, 34 were appropriate for the tasks, 27 were diverse (unique) and 30 were novel. 


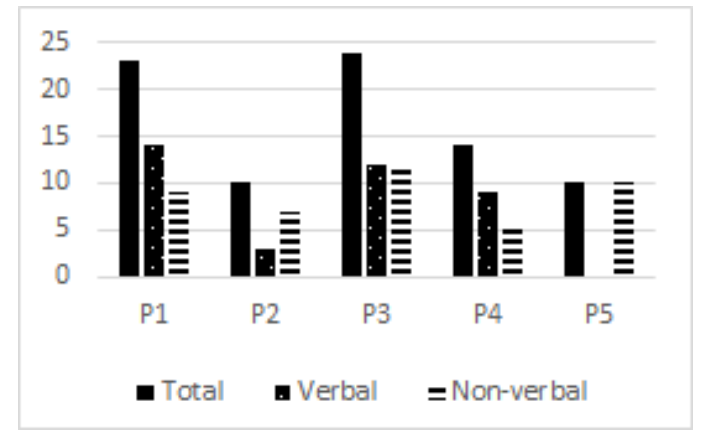

Figure 7. Instances of enjoyment with the prototype

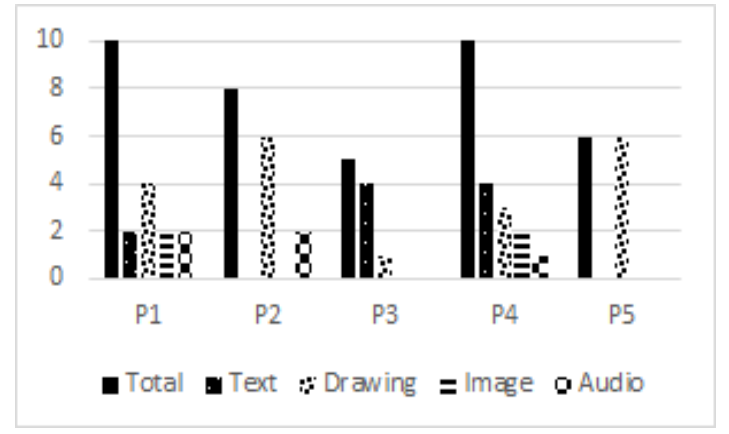

Figure 8. Numbers of ideas per child

\section{DISCUSSION}

We investigated the feasibility of a future technology-based tool (iGeneration), specifically designed to support autistic children to engage and contribute their ideas during PD's idea generation, as individually in this initial version. We employed a low fidelity prototype which had been iteratively designed and improved based on a series of studies involving 20 stakeholders. Our main aims were to find out whether children with autism would engage with the prototype, enjoy working with it and be capable of generating design ideas.

The fact that all participants completed the tasks without asking for a break and spent on average a very high percentage (96\%), and a minimum of $89 \%$, of session time on them indicates that the tool is promising for engaging children with autism during the idea generation stage of PD. Both the results from questionnaires and behaviour observations (verbal and non-verbal) clearly indicate that the children enjoyed using the prototype. The child who selected the emoticon 'neither happy nor unhappy', when asked for a reason for her answer, explained that she would like the prototype to be on a tablet. This suggests that she would enjoy more using a more finished product, rather than not liking the prototype at all. The small variability of opinions on ways of recording design ideas may be related to each child's abilities, and it would be interesting to further investigate how these features could be made more useful and attractive to children. Children were all able to generate ideas (at least one per task) and four out of the five generated more than one idea for at least one task. With few exceptions, the ideas were appropriate to the task. Sometimes the children were distracted, e.g. one of them (P3) was interested in Microsoft products and spent a long time describing them, and another (P2) appeared to sometimes be more interested in drawing than relating the idea to the task. Designing features to redirect child attention to the task as well as taking advantage of their interests to facilitate idea generation should be considered at the next stage of the tool design. In addition, most of the ideas generated were quite diverse (not repetitive) and novel (with no hint needed before).

Although our findings are promising, there are a few limitations which should be considered in the future. Firstly, our sample size was small, therefore the reported findings should be interpreted with caution. Actually, most studies with children with ASC include small sample sizes, mainly due to a general difficulty in recruiting participants (Bozgeyikli et al. 2018). However, in spite of the small sample, this study has contributed to our understanding of how a new technology-based tool can support children with ASC to contribute their ideas during a PD process. Moreover, children's suggestions will help us further improve the design of the tool. Secondly, our study had a time limit which was imposed by the autistic organisation's holiday campus activities. After developing the high-fidelity prototype, we intend to conduct a within-subject design study with children with ASC to compare engagement, enjoyment and ideas generated (both in quantity and quality) when working with it (condition A) and without (condition B). In addition, we are interested to look at the impact of the tool on children's behaviour and abilities (e.g. anxiety, self-efficacy).

Despite the limitations of this study, the results support a positive answer for the research questions, and constitute a strong basis for us to continue designing and developing iGeneration. In version two of iGeneration, we will focus on including collaborative idea generation (i.e. children working together to generate design ideas) and automatic personalised hints to support autistic children. For the latter, an idea 
reported in (Constantin et al. 2019) is to use crowdsourcing with a team of selected online workers (experts and practitioners in autism) as a way of generating hints and rating them in terms of appropriateness and usefulness for a child. Machine learning could remove noise and redundancy before the most frequent, appropriate and useful hint is provided. This approach has ethical challenges which will need to be addressed. We will also consider personalising other features for autistic children (e.g. the VC, the rewards).

\section{REFERENCES}

American Psychiatry Association (APA). 2013. Diagnostic and Statistical Manual of Mental Disorders Fifth Edition DSM-V. Washington, DC.

Braun, V. and Clarke, V., 2006. Using thematic analysis in psychology. Qualitative research in psychology, 3(2): 77-101.

Constantin, A., Alexandru, C.A. and Dragomir, M., 2019. Using crowdsourcing to foster creativity in children with autism during Idea Generation. CHI'19 Extended Abstracts.

Constantin, A. and Hourcade, J.P., 2018, October. Toward a technology-based tool to support Idea Generation during Participatory Design with children with Autism Spectrum Disorders. In Proceedings of the 20th International ACM SIGACCESS Conference on Computers and Accessibility, 385-387.

Constantin, A., Johnson, H., Smith, E., Lengyel, D., \& Brosnan, M., 2017. Designing computer-based rewards with and for children with Autism Spectrum Disorder and/or Intellectual Disability. Computers in Human Behav., 75: 404-414.

Constantin, A., Korte, J., Fails, J.A., Good, J., Alexandru, C.A., Dragomir, M., Pain, H., Hourcade, J.P., Eriksson, E., Waller, A. and Garzotto, F., 2019, June. Pushing the boundaries of participatory design with children with special needs. In Proceedings of the 18th ACM International Conference on Interaction Design and Children, 697-705.

De Leo, G. and Leroy, G., 2008, June. Smartphones to facilitate communication and improve social skills of children with severe autism spectrum disorder: special education teachers as proxies. In Proceedings of the 7th international conference on Interaction design and children, 45-48.

Druin, A., 2002. The role of children in the design of new technology. Behaviour and information technology, 21(1):1-25.

Escobedo, L., Tentori, M., Quintana, E., Favela, J. and Garcia-Rosas, D., 2014. Using augmented reality to help children with autism stay focused. IEEE Pervasive Computing, 13(1):38-46.

Fails, J.A., Guha, M.L. and Druin, A., 2013. Methods and techniques for involving children in the design of new technology for children. Foundations and Trends in Human-Computer Interaction, 6(2):85-166.

Frauenberger, C., Good, J., Alcorn, A. and Pain, H., 2013. Conversing through and about technologies: Design critique as an opportunity to engage children with autism and broaden research (er) perspectives. IJCCI, 1(2):38-49.

Grawemeyer, B., Johnson, H., Brosnan, M., Ashwin, E. and Benton, L., 2012, June. Developing an embodied pedagogical agent with and for young people with autism spectrum disorder. In International Conference on Intelligent Tutoring Systems, 262-267. Springer, Berlin, Heidelberg.

Iversen, O.S., Smith, R.C. and Dindler, C., 2017, June. Child as protagonist: Expanding the role of children in participatory design. In Proceedings of the 2017 Conference on Interaction Design and Children, 27-37.

Keay-Bright, W., 2007. The reactive colours project: demonstrating participatory and collaborative design methods for the creation of software for autistic children. Design Principles and Practices: an International Journal, 1(2): 7-15.

Malinverni, L., Mora-Guiard, J., Padillo, V., Mairena, M., Hervás, A. and Pares, N., 2014, June. Participatory design strategies to enhance the creative contribution of children with special needs. In Proceedings of the 2014 conference on Interaction design and children, 85-94.

Mazzone, L., Ruta, L. and Reale, L., 2012. Psychiatric comorbidities in asperger syndrome and high functioning autism: diagnostic challenges. Annals of general psychiatry, 11(1):16.

Randolph, J.J. and Eronen, P.J., 2007. Developing the Learning Door: A case study in youth participatory program planning. Evaluation and Program Planning, 30(1):55-65.

Read, J.C. and MacFarlane, S., 2006, June. Using the fun toolkit and other survey methods to gather opinions in child computer interaction. In Proceedings of the 2006 conference on Interaction design and children, 81-88.

Ropar, D. and Peebles, D., 2007. Sorting preference in children with autism: the dominance of concrete features. Journal of autism and developmental disorders, 37(2), 270-280.

Xie, L., Antle, A.N. and Motamedi, N., 2008, February. Are tangibles more fun? Comparing children's enjoyment and engagement using physical, graphical and tangible user interfaces. In Proceedings of the 2 nd international conference on Tangible and embedded interaction, 191-198. 